Lynton Graetz ${ }^{1,2}$ Lynley Bradnam ${ }^{1,3 *}$

'Applied Brain Research Laboratory, Centre for Neuroscience, School of Medicine

Flinders University, Adelaide, Australia

\section{${ }^{2}$ School of Psychology, Flinders University, Adelaide, Australia}

${ }^{3}$ Effectiveness of Therapy Group, Centre for Clinical Change and Healthcare Research, School of Medicine, Flinders University, Adelaide, Australia

Received 07 November 2013 accepted 22 November 2013

\title{
NON-INVASIVE CEREBELLAR STIMULATION IN DYSTONIA
}

\section{Abstract}

Primary isolated dystonia is a hyperkinetic movement disorder whereby involuntary muscle contractions cause twisted and abnormal postures. Dystonia of the cervical spine and upper limb may present as sustained muscle contractions or task-specific activity when using the hand or upper limb. There is little understanding of the pathophysiology underlying dystonia and this presents a challenge for clinicians and researchers alike. Emerging evidence that the cerebellum is involved in the pathophysiology of dystonia using network models presents the intriguing concept that the cerebellum could provide a novel target for non-invasive brain stimulation. Non-invasive stimulation to increase cerebellar excitability improved aspects of handwriting and circle drawing in a small cohort of people with focal hand and cervical dystonia. Mechanisms underlying the improvement in function are unknown, but putative pathways may involve the red nucleus and/or the cervical propriospinal system. Furthermore, recent understanding that the cerebellum has both motor and cognitive functions suggests that non-invasive cerebellar stimulation may improve both motor and non-motor aspects of dystonia. We propose a combination of motor and non-motor tasks that challenge cerebellar function may be combined with cerebellar non-invasive brain stimulation in the treatment of focal dystonia. Better understanding of how the cerebellum contributes to dystonia may be gained by using network models such as our putative circuits involving red nucleus and/or the cervical propriospinal system. Finally, novel treatment interventions encompassing both motor and non-motor functions of the cerebellum may prove effective for neurological disorders that exhibit cerebellar dysfunction.

Keywords

- Propriospinal $\cdot$ Red nucleus $\cdot$ Rubrospinal $\cdot$ Transcranial direct current stimulation $\cdot$ Non-motor

- Focal hand dystonia $\cdot$ Cervical dystonia

(c) Versita Sp. z o.o.

usually appears between the ages of 40 and 60 years as focal hand dystonia (FHD) or cervical dystonia (CD). CD has a significant effect on quality of life $[2,3]$ and the standard treatment at present is botulinum toxin injections into affected muscles [4]. Physiotherapy based treatments aimed at retraining posture or stretching dystonic muscles are also largely ineffective $[5,6]$. More recently, deep brain stimulation targeting the globus pallidus of the basal ganglia has shown promising results [7-10]. Efforts to discover effective non-invasive treatments have led researchers to non-invasive brain stimulation (NBS). Research to date has highlighted abnormalities in the function of inhibitory circuits within the primary motor cortex (M1) [11], whereby there is over-activity as measured by functional magnetic resonance imaging even during tasks that do not trigger dystonic postures [12]. Effects of non-invasive brain stimulation (NBS) to suppress M1 activity, either directly [13-15] or via premotor-M1 connections $[16,17]$ have had mixed results to date. However, evidence of a wide network of brain dysfunction in the pathophysiology of dystonia that include the cerebellum [18-22], opens up the prospect of applying NBS to these novel targets. Furthermore, emerging evidence that the cerebellum is highly involved in moderating certain aspects of cognition $[23,24]$ suggest that stimulating both motor and non-motor functions in therapeutic interventions may improve cerebellar function in neurological disorders like dystonia. The circuitry underlying benefits of cerebellar NBS for the treament of dystonia is uncertain. In this paper we present two putative network models to explain how cerebellar NBS may improve function of the upper limb and hand in cervical and upper limb dystonia. The first is a cerebello- with an uncertain aetiology [1]. The most frequent presentation, isolated focal dystonia

*E-mail: Lynley.bradnam@flinders.edu.au 
red nuclear-rubrospinal pathway, and the second is a cerebello-brainstem-propriospinal pathway. The implications of novel treatment paradigms that target cerebellar function in primary isolated dystonia are discussed.

\section{The cerebellum and dystonia}

There is a rapidly expanding body of research in rodent models that support a role for the cerebellum in the pathophysiology of primary dystonia [25], and indicates dysfunction at the level of Purkinje cells in the cerebellar cortex $[26,27]$. Evidence from basic neuroscience is supported by studies in humans. A postmortem examination of brains from people who had experienced CD demonstrated local loss of inhibitory Purkinje neurons from the cerebellar cortex $[28,29]$. Furthermore and neuroimaging studies show both functional and structural abnormalities in cerebellum and white matter tracts projecting from cerebellum to motor cortex [30]. Evidence from people with secondary dystonia caused by cerebellar lesions supports this thinking [31]. Strong neurophysiological evidence comes from a study using the conditioned the eye blink reflex, a paired associative learning paradigm that relies on inferior olivo-cerebellar circuits. Eye blink conditioning can be disrupted in healthy adults by non-invasive stimulation of the cerebellum [32]. Furthermore, people with CD have difficulty in developing the conditioned eye blink response [33], but cerebellar NBS improved their ability to develop conditioned eye blinks [34]. The ability to reverse a functional deficit in cerebellar processing indicates cerebellar NBS may provide a novel treatment target. Our own studies have found benefits for handwriting following cerebellar NBS in a small cohort of patients in support of this idea [35].

The extent of cerebellar modulation of M1 excitability in humans can be assessed using transcranial magnetic stimulation (TMS). The cerebellum imposes a level of tonic inhibition over M1 via the cerebello-thalamo-cortical pathway, which can be investigated using paired-pulse TMS [36-38]. A weak conditioning magnetic pulse over the cerebellum suppresses the magnitude of a second pulse applied to M1, known as cerebellar-brain inhibition (CBI). The tonic inhibitory tone imposed by $\mathrm{CB}$ is removed at the onset of a hand muscle contraction in healthy adults in a non-specific manner [39]. The usual cerebellar inhibitory modulation of $M 1$ is reduced in patients with FHD, which may contribute to the abnormal co-contraction of hand muscles during fine motor tasks [40]. Our own research found there is tonic $\mathrm{CBI}$ in people with $\mathrm{FHD}$ and $\mathrm{CD}$, however $\mathrm{CBI}$ is only evoked by a stronger magnetic simulation applied to the cerebellum compared to healthy controls [35]. This finding indicates the cerebellum may be underactive in patients, in agreement with findings from functional magnetic resonance imaging studies in people with dystonia [21] A second TMS technique to study cerebellar modulation of $\mathrm{M} 1$ is known as cerebellar paired associative stimuli (PAS) [41]. Pairing TMS of cerebellum and M1 at selected timing intervals evokes bidirectional changes in $\mathrm{M} 1$ activity in healthy adults. Short-interval pulses increase M1 excitability and long-interval pulses decrease $\mathrm{M} 1$ excitability. Pilot testing in our laboratory indicates the inhibitory effect at longer PAS intervals may be reduced in people with CD (L. Bradnam, unpublished data). As a result, a hypoactive cerebellum may not inhibit $\mathrm{M} 1$ representations of affected muscles appropriately and this might contribute to motor deficits in FHD and CD.

We recently showed that NBS of the cerebellum in the form of anodal transcranial direct current stimulation (TDCS) improved some kinematics of handwriting and cyclic drawing tasks in people with FHD and CD [35]. In an attempt to elucidate the mechanisms underlying the improvements in motor control, we measured $\mathrm{CBI}$ before and after cerebellar TDCS. There was a reduction in CBI after anodal TDCS; however there was no correlation with the improvement in task performance as measured by kinematic analysis of handwriting and cyclic drawing. This led us to consider alternative pathways for how cerebellar TDCS may have influenced handwriting in these people. The neuroanatomy of the cerebellar output pathways provides a number of possibilities due to the extensive connections in the brainstem that include the red nucleus, reticular nuclei, superior colliculus, basal ganglia and trigeminal sensory motor nucleus [22]. Activity in cerebellar-brainstem circuits cannot be measured directly using TMS in humans and may explain why there was no association between the effect of cerebellar NBS on CBI and handwriting and circle-drawing tasks in our pilot study.

\section{Neuroanatomy of the cerebellum}

To propose a new network model to explain our results it is necessary to briefly review the anatomical connections of the cerebellum. Broadly, the cerebellum is divided into functional regions based on sources of sensory input. The cerebrocerebellum receives projections from the parietal, sensory and motor cortices via the pontine middle cerebellar peduncle. The vestibulocerebellum processes vestibular nuclei and superior colliculus input, while the spinocerebellum receives somatosensory information from the body via the spinocerebellar tracts and the inferior olive. The cerebellar cortex contains the inhibitory purkinje cells, the main output projections to the deep cerebellar nuclei, and other excitatory (granule) and inhibitory (basket) neurons and interneurons that modulate purkinje cell activity. Purkinje cells project to the deep cerebellar nuclei in a somatotopic fashion. Outputs from the deep cerebellar nuclei are conveyed by the superior cerebellar peduncle. The vestibulocerebellum projects to the vestibular nuclei giving rise to the descending vestibulospinal tracts and the superior colliculus, the origin of the tectospinal pathway. The fastigal and interposed nuclei (IN) project to the reticular nuclei and the red nucleus that respectively give rise to the reticulospinal and rubrospinal tracts. The outermost deep cerebellar nuclei, the dentate nucleus projects to premotor cortex and M1 via the thalamus and contributes to motor planning and descending output via the corticospinal tract. Finally, there are reciprocal projections between the cerebellum and the basal ganglia supporting modulation of basal ganglia motor output to the thalamus [42-44]. Abnormal function of the cerebellum can influence function of the basal ganglia and 
vice versa due to these reciprocal projections in the pathophysiology of dystonia [22]. In conclusion, dysfunction of the cerebellum can, via its anatomical connections, influence descending control over spinal motoneurons innervating cervical and upper limb muscles via several 'loops' that include cortical, brainstem and basal ganglia. These likely all contribute to the network model hypothesis, but we propose two putative pathways to explain our results of cerebellar TDCS on handwriting in people with dystonia.

\section{The cerebello-red nuclear- rubrospinal tract}

The IN of the deep cerebellar nuclei has vast excitatory projections to regions of the brainstem, including the contralateral red nucleus [45]. The IN is known to be involved in the generation and shaping of ipsilateral upper limb movement during reaching [46]. Somatotopically organised, the IN controls aspects of the reach to grasp manoeuvre, adaptation to perturbations during reaching and the precision of hand placement during reaching $[47,48]$. The red nucleus is the most direct pathway for the IN to influence activity in spinal motoneurons and interneurons innervating hand muscles [46] (Figure 1). The descending rubrospinal tract terminates mostly onto interneurons across the cervical spinal cord, but there are a small number of rubrospinal terminations onto lateral alphamotoneurons in the lower cervical cord to control fine finger movement [49-51]. In particular, the rubrospinal tract is important for coordinating upper limb muscles when reaching involves positioning the fingers for grasping [46,52] as in during the performance of handwriting. Consistent with this, lesions of the IN were found to impair the performance of multi-segmental movements of the upper limb [53]. The pattern of indirect rubrospinal projections to spinal interneurons across the cervical spine to innervate proximal upper limb muscles and direct projections to alphamotoneurons innervating finger muscles may serve to integrate motor control of the proximal and distal segments for coordinated muscle synergies [54]. If there is dysfunction in the cerebello-red nuclear-rubrospinal tract, this coordinated action of the hand and proximal upper limb would be degraded, partially explaining the motor dysfunction in the hand and upper limb in many presentations of isolated dystonia. Furthermore, since red nucleus projects to the upper cervical alphamotoneurons, facial motor nucleus $[55,56]$ and to the trigeminal sensory nuclear complex with direct connections to the trigeminal motor nucleus [57] the cerebellar-red nucleus circuitry could potentially explain the pathological activation of facial and neck muscles in $C D$ and craniofacial dystonia too (Figure 1).

\section{The cerebello-brainstem- propriospinal pathway}

The cerebellum could also influence activity in muscles controlling the hand by indirect modulation of projections to the $\mathrm{C} 3 / 4$ cervical propriospinal system [58] (Figure 2). The descending rubrospinal, reticulospinal and corticospinal tracts are all indirectly under cerebellar control and project to cervical propriospinal neurons (PN) and the local inhibitory interneurons that modulate their excitability $[59,60]$. Cervical propriospinal neurons (PN) are heavily involved in controlling upper limb reaching as PNs project to multiple motoneurons innervating forearm and hand muscles activated synergistically during reach to grasp tasks $[61,62]$. Cervical PN integrate sensory feedback from the hand and forearm with descending cortical drive to stabilise proximal muscles during use of the hand in reaching tasks [63-65]. Abnormal activity of cervical PN may play a role in the generation of aberrant muscle synergies of the hand and arm in dystonia by failing to suppress motoneurons innervating muscles not required for a particular task [66,67]. Propriospinal neurons provide critical feedback to the cerebellum regarding the state of descending motor commands from $\mathrm{M} 1$ and ascending peripheral afference from the forearm and hand. This integrative information processed within PN is relayed directly to the cerebellum via the lateral reticular nucleus $[68,69]$. In this manner, the cerebellum is provided with accurate

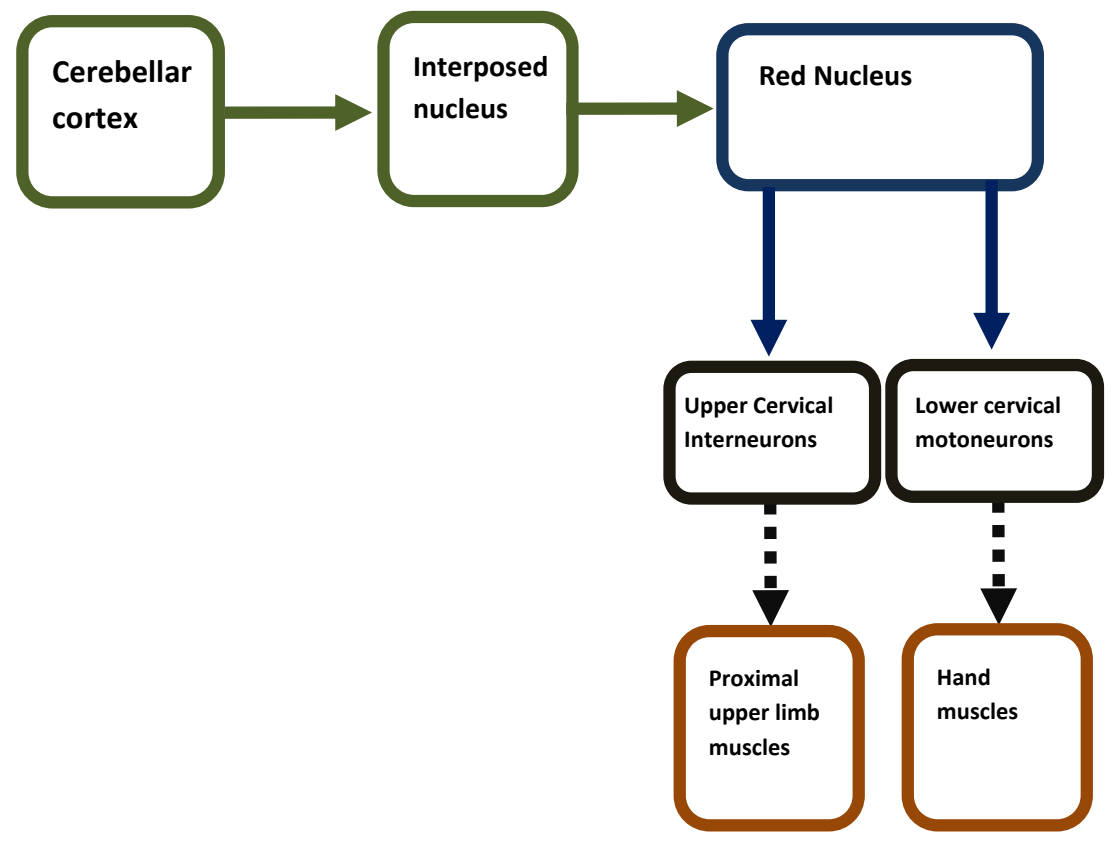

Figure 1. A simplified schematic illustrating the putative cerebello-red nuclear-rubrospinal pathway. The pathway involves projections from the cerebellar cortex to the interposed nucleus and from the interposed nucleus to the red nucleus. The red nucleus has descending projections via the rubrospinal tract to spinal interneurons at the upper and mid cervical levels and direct projections to lateral alpha-motoneurons in the lower cervical cord innervating hand muscles. The interposed nucleus via the rubrospinal tract controls reaching movements when the hand is positioned for grasping or fine manipulation. 


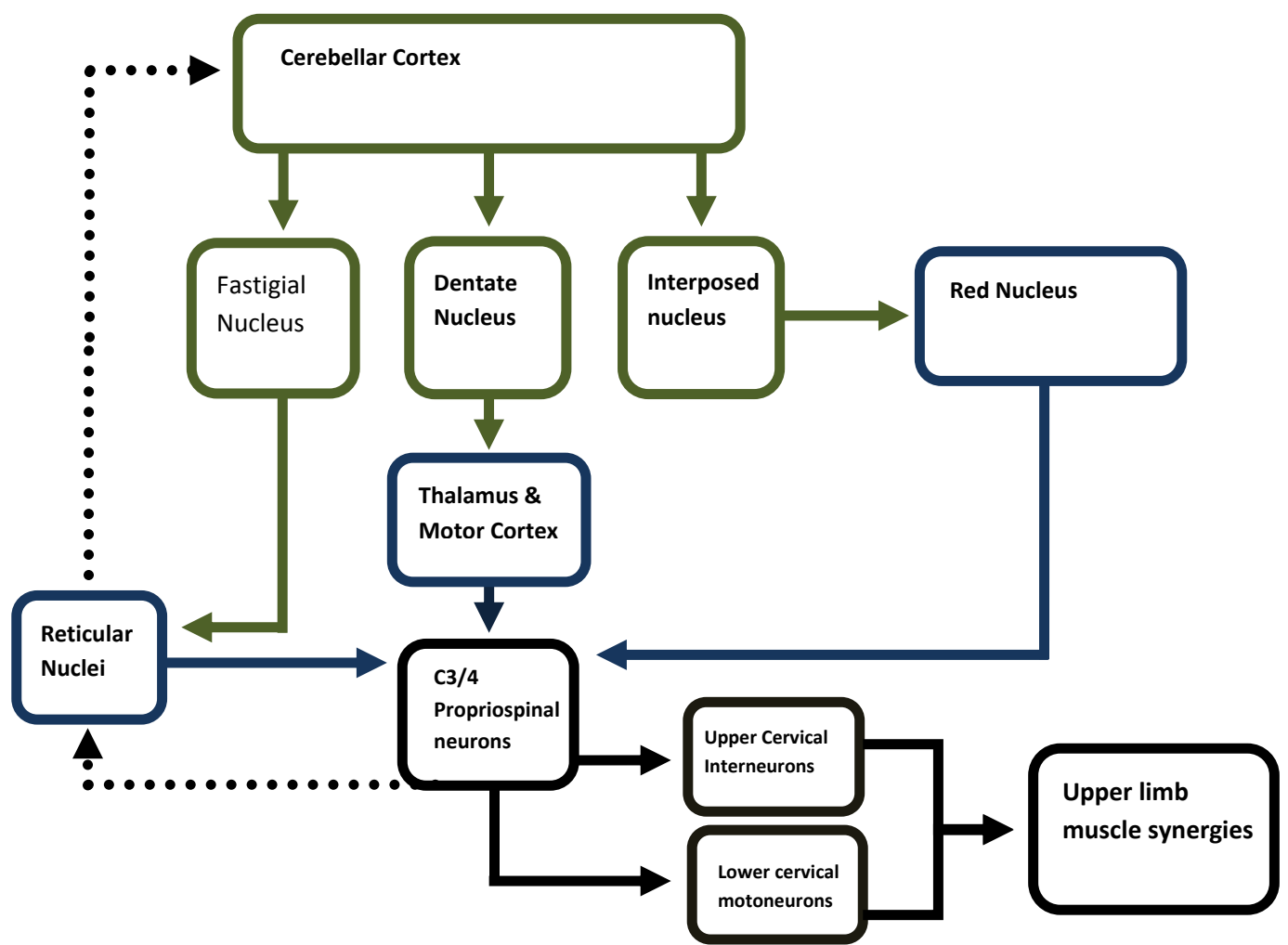

Figure 2. A simplified schematic illustrating the putative cerebello-brainstem-propriospinal pathway. The cerebellar cortex controls activity in C4/5 propriospinal neurons by outputs to the interposed nucleus - red nucleus projection and the fastigial - reticular nuclei projection. Both the red nucleus and the reticular nuclei have descending control over propriospinal neuron excitability via the rubrospinal and reticulospinal tracts respectively. The output projection from the dentate nucleus to motor cortex (cerebello-thalamo-cortical) that controls activity in propriospinal neurons via the descending corticospinal tract is also shown. Afferent feedback from the propriospinal neurons reaches the cerebellum via the lateral reticular nucleus, illustrated by the dashed arrows. The position of the deep cerebellar nuclei in relationship to one another is not anatomically correct.

information to update descending commands via $\mathrm{M} 1$ and subcortical projections to the spinal cord when required. In dystonia it is possible that the cerebellum does not recognise when feedback from PN is aberrant or incorrect for a particular reaching and grasping task.

The cervical PN system can be studied in humans using paired TMS and peripheral nerve stimulation [64]. At different intensities of peripheral nerve stimulation, both excitatory and inhibitory inputs to PNs can be tested. Using these techniques, TDCS over primary motor cortex was found to modulate both excitatory and inhibitory projections to cervical PNs [70], presumably by modulation of ipsilateral reticulospinal and rubrospinal tracts. Paired TMS and peripheral nerve stimulation to test cervical PN may provide an indirect method to test effects of brainstem contribution to dystonia that can't be tested using techniques such as CBI. Cervical PNs are involved in the selective activation and suppression of muscles as required for a particular functional synergy by their functional connections with many motoneurons innervating muscles across the limb. Selective muscle activation can be tested using another TMS paradigm, whereby motor-evoked responses of the biceps brachii are facilitated during elbow flexion and suppressed during forearm pronation to provide a 'selectivity ratio' [71-73]. Suppression of primary motor cortex excitability using NBS disrupted selective muscle activation in the ipsilateral arm in healthy adults, most likely by modulation of ipsilateral descending projections [71,73]. Studies investigating whether NBS to cerebellum influences sensori-motor integration in cervical PN and degrades or improves selective muscle activation in healthy adults and in people with FHD and CD may support our hypothesis of a cerebellar-brainstem-propriospinal pathway that contributes to the pathogenesis of dystonia. Such novel studies may provide better understanding of the pathways by which cerebellum modulates descending motor control to motoneurons innervating muscles affected by dystonia and the effects of cerebellar TDCS.

\section{The non-motor role of the cerebellum in dystonia}

The cerebellum encodes important movement parameters such as direction and speed of movement and the position of the limb [74] along with the timing and sequencing of movement [75]. There is a large contribution to motor learning, visuomotor adaptation and learning of new action sequences [76-78]. Nonmotor cerebellar functions are now receiving more attention with the improved awareness of cerebellar involvement in neurological 
conditions like dystonia and Parkinson's disease $[79,80]$. The cerebellum in important for procedural learning [81] and for spatial and verbal aspects of working memory [78]. In patients with cerebellar atrophy there is an impaired capacity for procedural learning, specifically when performing tasks with the hand ipsilateral to the side of the cerebellar lesion [82]. Procedural learning assessed by the serial reaction time test (SRTT) can be improved in healthy adults by cerebellar TDCS $[81,83]$. Several studies have found the cathodal TDCS to the cerebellum affected the performance of working memory tasks [84-86]. Finally, performance on a visuomotor adaptation task in healthy adults was improved by anodal TDCS [76].

We are interested in whether interventions that target cerebellum for motor learning may affect non-motor function and if promotion of nonmotor cerebellar learning may have benefits for motor control. Evidence for cognitive dysfunction in dystonia is still emerging. It is know that aspects of sensory and motor timing related to movement processing and planning are affected are disrupted in people with FHD [87]. There is emerging evidence that motor learning is deficient in people with cervical dystonia using neurophysiological measures of learning $[88,89]$. People with FHD are slower to adapt to visuomotor perturbations than healthy adults [90]. The only working memory task tested to date to our knowledge is the mental rotation task, a visuospatial working memory task [78]. People with FHD and $C D$ perform poorly on mental rotation tasks involving corporal objects [91,92]. Other aspects of procedural learning and working memory need to be systematically tested in people with dystonia along with the effect of cerebellar NBS on these cognitive processes. Another interesting aspect arising from a cerebellar contribution to cervical dystonia is that due to lateralisation in cerebellar function [81], there may be an association between deficits and the direction of head turn (i.e. dystonic posture). Lateralisation of the cerebellum is due to the mostly contralateral nature of the connections between cerebellum and cerebral cortex [93], although about 10\% of fibres project to the ipsilateral M1. The right cerebellum is concerned with linguistic processes while the left is specific to spatial information [94]. The left cerebellum has a greater role in visuospatial processing and in spatial working memory [78]. The right cerebellum is more associated with verbal working memory particularly when tested using a digit span working memory task [85] or the Paced Auditory Serial Subtraction Task (PASST) evaluating working memory and attention [86]. We have observed a tendency for left cerebellar stimulation to increase the randomness of responses in a random number generation task compared to right cerebellar stimulation and sham stimulation in healthy adults (L. Bradnam, unpublished results). The effect of neck rotation direction on motor and non-motor performance has not been systematically investigated. We have observed a trend in CD for those with right neck rotation to better recognise right neck rotation that was not apparent in those with left neck rotation. We have also observed that people with $C D$ are less likely to verbally generate numbers randomly, but we are yet to elucidate whether the side of neck rotation influences performance in this task (L. Bradnam unpublished results). Lateralisation of mental performance dependent on head turn requires more systematic investigation as it may provide key information toward individualising interventions in patients based on clinical tests.

\section{Implications for non-invasive brain stimulation to cerebellum}

NBS to cerebellum is a safe intervention [95] and early indications is that it may be effective for improving handwriting function in people with CD and FHD [35]. Effects of NBS may be augmented by task-specific motor training [96] and/or non-motor tasks that challenge known cerebellar functions [23]. Together, these therapies may provide an alterantive treatment for the painful muscle spasms and twisted neck postures, and the lesser known cognitive deficits such as sequencing and timing error that are associated with isolated dystonia. In addition to the potential physiological benefit, TDCS is a particularly practical technique for use in people with $C D$, because it does not require one's head to remain still, thus not applying additional stress to the impaired neck. Furthermore, it is easy to incorporate motor and non-motor training regimes concurrently with TDCS. However, there are still gaps in the knowledge base that impede the translation of TDCS into clinical practice. While it is recognised that repeated sessions of non-invasive brain stimulation are required to promote synaptic plasticity and learning in the human brain [97] many of the parameters of stimulation remain unclear. Issues such as optimum electrode placement and length and frequency of stimulation for best therapeutic effect remain to be elucidated.

\section{Conclusion}

We recently found anodal TDCS to cerebellum improved handwriting [35], potentially by modulation of a putative cerebello-red nuclearrubrospinal tract or a cerebello-brainstempropriospinal tract. Our results do not seem to be associated with changes in excitability of the cerebello-thalamo-cortical pathway. With the emergence of known non-motor functions of the cerebellum there may be a case for a 'multimodal approach' to treatment of dystonia. Multi-modal interventions would combine cerebellar TBS with individualised therapy delivered by task practice that challenges motor and non-motor learning and sequencing [23]. The on-going work in our laboratory take this approach to studies using cerebellar NBS in people with cervical dystonia. The aim is to provide evidence for the efficacy of these novel treatment paradigms utilising cerebellar NBS and cognitive and motor training to provide alternative, non-invasive treatments for people with these recalcitrant movement disorders.

\section{Acknowledgements}

The authors declare no conflict of interest. These experiments were funded by the Brain Foundation Australia. 
[1] Phukan J., Albanese A., Gasser T.,Warner T., Primary dystonia and dystonia-plus syndromes: clinical characteristics, diagnosis, and pathogenesis, Lancet Neurol., 2011, 10, 1074-1085

[2] Lim V.K., Health related quality of life in patients with dystonia and their caregivers in New Zealand and Australia, Mov. Disord., 2007, 22, 998-1003

[3] Zetterberg L., Aquilonius S.M., Lindmark B., Impact of dystonia on quality of life and health in a Swedish population, Acta Neurol. Scand., 2009, 119, 376-382

[4] Batla A., Stamelou M.,Bhatia K.P., Treatment of focal dystonia, Curr. Treat. Options Neurol., 2012, 14, 213-229

[5] Tassorelli C., Mancini F., Balloni L., Pacchetti C., Sandrini G., Nappi G., et al., Botulinum toxin and neuromotor rehabilitation: An integrated approach to idiopathic cervical dystonia, Mov. Disord., 2006, 21, 2240-2243

[6] Zetterberg L., Halvorsen K., Farnstrand C., Aquilonius S.M., Lindmark B., Physiotherapy in cervical dystonia: six experimental single-case studies, Physiother. Theory Pract., 2008, 24, 275-290

[7] Jahanshahi M., Torkamani M., Beigi M., Wilkinson L., Page D., Madeley L., et al., Pallidal stimulation for primary generalised dystonia: effect on cognition, mood and quality of life, J. Neurol., 2013, [Epub ahead of print], doi: 10.1007/s00415-013-7161-2

[8] Petrossian M.T., Paul L.R., Multhaupt-Buell T.J., Eckhardt C., Hayes M.T., Duhaime A.C., et al., Pallidal deep brain stimulation for dystonia: a case series, J. Neurosurg. Pediatr., 2013, [Epub ahead of print], doi: 10.3171/2013.8.PEDS13134

[9] Sadnicka A., Kimmich O., Pisarek C., Ruge D., Galea J., Kassavetis P., et al., Pallidal stimulation for cervical dystonia does not correct abnormal temporal discrimination, Mov. Disord., 2013, 28, 1874-1877

[10] Witt J.L., Moro E., Ash R.S., Hamani C., Starr P.A., Lozano A.M., et al., Predictive factors of outcome in primary cervical dystonia following pallidal deep brain stimulation, Mov. Disord., 2013, 28, 1451-1455

[11] Hallett M., Neurophysiology of dystonia: the role of inhibition, Neurobiol. Dis., 2011, 42, 177-184

[12] Kimberley T.J.,Pickett K.A., Differential activation in the primary motor cortex during individual digit movement in focal hand dystonia vs. healthy, Restor. Neurol. Neurosci., 2012, 30, 247-254

[13] Benninger D.H., Lomarev M., Lopez G., Pal N., Luckenbaugh D.A.,Hallett M., Transcranial direct current stimulation for the treatment of focal hand dystonia, Mov. Disord., 2011, 26, 1698-1702

[14] Buttkus F., Baur V., Jabusch H.C., de la Cruz Gomez-Pellin M., Paulus W., Nitsche M.A., et al., Single-session tDCS-supported retraining does not improve fine motor control in musician's dystonia, Restor. Neurol. Neurosci., 2011, 29, 85-90

[15] Huang Y.Z., Rothwell J.C., Lu C.S., Wang J.,Chen R.S., Restoration of motor inhibition through an abnormal premotor-motor connection in dystonia, Mov. Disord., 2010, 25, 696-703

[16] Kimberley T.J., Borich M.R., Arora S., Siebner H.R., Multiple sessions of low-frequency repetitive transcranial magnetic stimulation in focal hand dystonia: clinical and physiological effects, Restor. Neurol. Neurosci., 2013, 31, 533-542

[17] Borich M., Arora S., Kimberley T.J., Lasting effects of repeated rTMS application in focal hand dystonia, Restor. Neurol. Neurosci., 2009, 27, 55-65

[18] Jinnah H.A., Hess E.J., A new twist on the anatomy of dystonia: the basal ganglia and the cerebellum?, Neurology, 2006, 67, 1740-1741

[19] Neychev V.K., Fan X., Mitev V.I., Hess E.J., Jinnah H.A., The basal ganglia and cerebellum interact in the expression of dystonic movement, Brain, 2008, 131, 2499-2509

[20] Neychev V.K., Gross R.E., Lehericy S., Hess E.J.,Jinnah H.A., The functional neuroanatomy of dystonia, Neurobiol. Dis., 2011, 42, 185-201

[21] Wu C.C., Fairhall S.L., McNair N.A., Hamm J.P., Kirk I.J., Cunnington R., et al., Impaired sensorimotor integration in focal hand dystonia patients in the absence of symptoms, J. Neurol. Neurosurg. Psychiatry, 2010, $81,659-665$

[22] Bradnam L., Barry C., The role of the trigeminal sensory nuclear complex in the pathophysiology of cranio-cervical dystonia, J. Neurosci., 2013, 33, 18358-18367

[23] Tedesco A.M., Chiricozzi F.R., Clausi S., Lupo M., Molinari M., Leggio M.G., The cerebellar cognitive profile, Brain, 2011, 134, 3672-3686

[24] Koziol L.F., Budding D., Andreasen N., D'Arrigo S., Bulgheroni S., Imamizu H., et al., Consensus paper: the cerebellum's role in movement and cognition, Cerebellum, 2013, [Epub ahead of print], 10.1007/s12311-013-0511-x

[25] Wilson B.K., Hess E.J., Animal models for dystonia, Mov. Disord., 2013, 28, 982-989

[26] Raike R.S., Pizoli C.E., Weisz C., van den Maagdenberg A.M., Jinnah H.A.,Hess E.J., Limited regional cerebellar dysfunction induces focal dystonia in mice, Neurobiol. Dis., 2012, 49C, 200-210

[27] Todorov B., Kros L., Shyti R., Plak P., Haasdijk E.D., Raike R.S., et al., Purkinje cell-specific ablation of Cav2.1 channels is sufficient to cause cerebellar ataxia in mice, Cerebellum, 2012, 11, 246-258

[28] Prudente C.N., Pardo C.A., Xiao J., Hanfelt J., Hess E.J., Ledoux M.S., et al., Neuropathology of cervical dystonia, Exp. Neurol., 2013, 241, $95-$ 104

[29] Zoons E., Tijssen M.A., Pathologic changes in the brain in cervical dystonia pre- and post-mortem - a commentary with a special focus on the cerebellum, Exp. Neurol., 2013, 247, 130-133

[30] Argyelan M., Carbon M., Niethammer M., Ulug A.M., Voss H.U., Bressman S.B., et al., Cerebellothalamocortical connectivity regulates penetrance in dystonia, J. Neurosci., 2009, 29, 9740-9747

[31] Doshi A., Rohrer J., Warner T., A case supporting the role of the cerebellum in dystonia, J. Neurol. Neurosurg. Psychiatry, 2013, 84, e2

[32] Hoffland B.S., Bologna M., Kassavetis P., Teo J.T., Rothwell J.C., Yeo C.H., et al., Cerebellar theta burst stimulation impairs eyeblink classical conditioning, J. Physiol., 2012, 590, 887-897

[33] Teo J.T., van de Warrenburg B.P., Schneider S.A., Rothwell J.C., Bhatia K.P., Neurophysiological evidence for cerebellar dysfunction in 
primary focal dystonia, J. Neurol. Neurosurg. Psychiatry, 2009, 80, 8083

[34] Hoffland B.S., Kassavetis P., Bologna M., Teo J.T., Bhatia K.P., Rothwell J.C., et al., Cerebellum-dependent associative learning deficits in primary dystonia are normalized by rTMS and practice, Eur. J. Neurosci., 2013, 38, 2166-2171

[35] Bradnam L., Graetz L., McDonnell M., Ridding M., Non-invasive cerebellar stimulation in focal dystonia, Mov. Disord., 2013, 28, S10

[36] Daskalakis Z.J., Paradiso G.O., Christensen B.K., Fitzgerald P.B., Gunraj C., Chen R., Exploring the connectivity between the cerebellum and motor cortex in humans, J. Physiol., 2004, 557, 689-700

[37] Ugawa Y., Uesaka Y., Terao Y., Hanajima R.,Kanazawa I., Magnetic stimulation over the cerebellum in humans, Ann. Neurol., 1995, 37, 703-713

[38] Oliveri M., Torriero S., Koch G., Salerno S., Petrosini L., Caltagirone C., The role of transcranial magnetic stimulation in the study of cerebellar cognitive function, Cerebellum, 2007, 6, 95-101

[39] Kassavetis P., Hoffland B.S., Saifee T.A., Bhatia K.P., van de Warrenburg B.P., Rothwell J.C., et al., Cerebellar brain inhibition is decreased in active and surround muscles at the onset of voluntary movement, Exp. Brain Res., 2011, 209, 437-442

[40] Brighina F., Romano M., Giglia G., Saia V., Puma A., Giglia F., et al., Effects of cerebellar TMS on motor cortex of patients with focal dystonia: a preliminary report, Exp. Brain Res., 2009, 192, 651-656

[41] Lu M.K., Tsai C.H., Ziemann U., Cerebellum to motor cortex paired associative stimulation induces bidirectional STDP-like plasticity in human motor cortex, Front. Hum. Neurosci., 2012, 6, 260

[42] Bostan A.C., Dum R.P., Strick P.L., The basal ganglia communicate with the cerebellum, Proc. Natl. Acad. Sci. USA, 2010, 107, 8452-8456

[43] Bostan A.C., Dum R.P., Strick P.L., Cerebellar networks with the cerebral cortex and basal ganglia, Trends Cogn. Sci., 2013, 17, 241-254

[44] Bostan A.C., Strick P.L., The cerebellum and basal ganglia are interconnected, Neuropsychol. Rev., 2010, 20, 261-270

[45] Teune T.M., van der Burg J., van der Moer J., Voogd J., Ruigrok T.J., Topography of cerebellar nuclear projections to the brain stem in the rat, Prog. Brain Res., 2000, 124, 141-172

[46] Perciavalle V., Apps R., Bracha V., Delgado-Garcia J.M., Gibson A.R., Leggio M., et al., Consensus paper: current views on the role of cerebellar interpositus nucleus in movement control and emotion, Cerebellum, 2013, 12, 738-757

[47] Bracha V., Kolb F.P., Irwin K.B., Bloedel J.R., Inactivation of interposed nuclei in the cat: classically conditioned withdrawal reflexes, voluntary limb movements and the action primitive hypothesis, Exp. Brain Res., 1999, 126, 77-92

[48] Martin J.H., Cooper S.E., Hacking A.,Ghez C., Differential effects of deep cerebellar nuclei inactivation on reaching and adaptive control, J. Neurophysiol., 2000, 83, 1886-1899

[49] Holstege G., Blok B.F., Ralston D.D., Anatomical evidence for red nucleus projections to motoneuronal cell groups in the spinal cord of the monkey, Neurosci. Lett., 1988, 95, 97-101

[50] Fujito Y., Aoki M., Monosynaptic rubrospinal projections to distal forelimb motoneurons in the cat, Exp. Brain Res., 1995, 105, 181-190
[51] Robinson F.R., Houk J.C., Gibson A.R., Limb specific connections of the cat magnocellular red nucleus, J. Comp. Neurol., 1987, 257, 553-577

[52] Gibson A.R., Horn K.M., Stein J.F., Van Kan P.L., Activity of interpositus neurons during a visually guided reach, Can. J. Physiol. Pharmacol., 1996, 74, 499-512

[53] Thach W.T., Goodkin H.P., Keating J.G., The cerebellum and the adaptive coordination of movement, Annu. Rev. Neurosci., 1992, 15, 403-442

[54] Mason C.R., Miller L.E., Baker J.F., Houk J.C., Organization of reaching and grasping movements in the primate cerebellar nuclei as revealed by focal muscimol inactivations, J. Neurophysiol., 1998, 79, 537-554

[55] Pong M., Horn K.M., Gibson A.R., Spinal projections of the cat parvicellular red nucleus, J. Neurophysiol., 2002, 87, 453-468

[56] Morcuende S., Delgado-Garcia J.M., Ugolini G., Neuronal premotor networks involved in eyelid responses: retrograde transneuronal tracing with rabies virus from the orbicularis oculi muscle in the rat, $\mathrm{J}$. Neurosci., 2002, 22, 8808-8818

[57] Satoh Y., Yajima E., Ishizuka K., Nagamine Y., Iwasaki S., Modulation of two types of jaw-opening reflex by stimulation of the red nucleus, Brain Res. Bull., 2013, 97, 24-31

[58] Pierrot-Deseilligny E., Burke D., The circuitry of the human spinal cord. Its role in motor control and movement disorders, Cambridge University Press, Cambridge, UK, 2005

[59] Alstermark B., Lundberg A., Sasaki S., Integration in descending motor pathways controlling the forelimb in the cat. 11. Inhibitory pathways from higher motor centres and forelimb afferents to C3-C4 propriospinal neurones, Exp. Brain Res., 1984, 56, 293-307

[60] Alstermark B., Lundberg A., Sasaki S., Integration in descending motor pathways controlling the forelimb in the cat. 10 . Inhibitory pathways to forelimb motoneurones via C3-C4 propriospinal neurones, Exp. Brain Res., 1984, 56, 279-292

[61] Alstermark B., Isa T., Circuits for skilled reaching and grasping, Annu. Rev. Neurosci., 2012, 35, 559-578

[62] Alstermark B., Gorska T., Johannisson T., Lundberg A., Hypermetria in forelimb target-reaching after interruption of the inhibitory pathway from forelimb afferents to C3-C4 propriospinal neurones, Neurosci. Res., 1986, 3, 457-461

[63] Iglesias C., Marchand-Pauvert V., Lourenco G., Burke D., PierrotDeseilligny E., Task-related changes in propriospinal excitation from hand muscles to human flexor carpi radialis motoneurones, J. Physiol., 2007, 582, 1361-1379

[64] Roberts L.V., Stinear C.M., Lewis G.N., Byblow W.D., Task-dependent modulation of propriospinal inputs to human shoulder, J. Neurophysiol., 2008, 100, 2109-2114

[65] Giboin L.S., Lackmy-Vallee A., Burke D., Marchand-Pauvert V., Enhanced propriospinal excitation from hand muscles to wrist flexors during reach-to-grasp in humans, J. Neurophysiol., 2012, 107, $532-543$

[66] Lourenco G., Bleton J.P., Iglesias C., Vidailhet M., Marchand-Pauvert V., Abnormal spinal interactions from hand afferents to forearm muscles in writer's cramp, Clin. Neurophysiol., 2007, 118, 2215-2226

[67] Marchand-Pauvert V., Iglesias C., Properties of human spinal 
interneurones: normal and dystonic control, J. Physiol., 2008, 586, 1247-1256

[68] Alstermark B., Isa T., Tantisira B., Projection from excitatory C3C4 propriospinal neurones to spinocerebellar and spinoreticular neurones in the C6-Th1 segments of the cat, Neurosci. Res., 1990, 8 , 124-130

[69] Alstermark B., Lindstrom S., Lundberg A.,Sybirska E., Integration in descending motor pathways controlling the forelimb in the cat. 8. Ascending projection to the lateral reticular nucleus from $\mathrm{C3}-\mathrm{C} 4$ propriospinal also projecting to forelimb motoneurones, Exp. Brain Res., 1981, 42, 282-298

[70] Bradnam L.V., Stinear C.M., Byblow W.D., Cathodal transcranial direct current stimulation suppresses ipsilateral projections to presumed propriospinal neurons of the proximal upper limb, J. Neurophysiol., $2011,105,2582-2589$

[71] Bradnam L.V., Stinear C.M., Byblow W.D., Theta burst stimulation of human primary motor cortex degrades selective muscle activation in the ipsilateral arm, J. Neurophysiol., 2010, 104, 2594-2602

[72] Gerachshenko T., Stinear J.W., Suppression of motor evoked potentials in biceps brachii preceding pronator contraction, Exp. Brain Res., 2007, 183, 531-539

[73] McCambridge A.B., Bradnam L.V., Stinear C.M., Byblow W.D., Cathodal transcranial direct current stimulation of the primary motor cortex improves selective muscle activation in the ipsilateral arm, J. Neurophysiol., 2011, 105, 2937-2942

[74] Apps R., Movement-related gating of climbing fibre input to cerebellar cortical zones, Prog. Neurobiol., 1999, 57, 537-562

[75] Leggio M.G., Chiricozzi F.R., Clausi S., Tedesco A.M., Molinari M., The neuropsychological profile of cerebellar damage: the sequencing hypothesis, Cortex, 2011, 47, 137-144

[76] Galea J.M., Vazquez A., Pasricha N., de Xivry J.J., Celnik P., Dissociating the roles of the cerebellum and motor cortex during adaptive learning: the motor cortex retains what the cerebellum learns, Cereb. Cortex, 2011, 21, 1761-1770

[77] Torriero S., Oliveri M., Koch G., Lo Gerfo E., Salerno S., Ferlazzo F., et al., Changes in cerebello-motor connectivity during procedural learning by actual execution and observation, J. Cogn. Neurosci., 2011, 23, 338-348

[78] Bernard J.A.,Seidler R.D., Cerebellar contributions to visuomotor adaptation and motor sequence learning: an ALE meta-analysis, Front. Hum. Neurosci., 2013, 7, 27

[79] Stamelou M., Edwards M.J., Hallett M., Bhatia K.P., The non-motor syndrome of primary dystonia: clinical and pathophysiological implications, Brain, 2012, 135, 1668-1681

[80] Wu T., Hallett M., The cerebellum in Parkinson's disease, Brain, 2013, 136, 696-709

[81] Torriero S., Oliveri M., Koch G., Caltagirone C., Petrosini L., Interference of left and right cerebellar rTMS with procedural learning, J. Cogn. Neurosci., 2004, 16, 1605-1611

[82] Torriero S., Oliveri M., Koch G., Lo Gerfo E., Salerno S., Petrosini L., et al.,
Cortical networks of procedural learning: evidence from cerebellar damage, Neuropsychologia, 2007, 45, 1208-1214

[83] Ferrucci R., Brunoni A.R., Parazzini M., Vergari M., Rossi E., Fumagalli M., et al., Modulating human procedural learning by cerebellar transcranial direct current stimulation, Cerebellum, 2013, 12, 485-492

[84] Ferrucci R., Marceglia S., Vergari M., Cogiamanian F., Mrakic-Sposta S., Mameli F., et al., Cerebellar transcranial direct current stimulation impairs the practice-dependent proficiency increase in working memory, J. Cogn. Neurosci., 2008, 20, 1687-1697

[85] Boehringer A., Macher K., Dukart J., Villringer A., Pleger B., Cerebellar transcranial direct current stimulation modulates verbal working memory, Brain Stimul., 2013, 6, 649-653

[86] Pope P.A., Miall R.C., Task-specific facilitation of cognition by cathodal transcranial direct current stimulation of the cerebellum, Brain Stimul., 2012, 5, 84-94

[87] Avanzino L., Martino D., Martino I., Pelosin E., Vicario C.M., Bove M., et al., Temporal expectation in focal hand dystonia, Brain, 2013, 136, 444-454

[88] Meunier S., Russmann H., Shamim E., Lamy J.C., Hallett M., Plasticity of cortical inhibition in dystonia is impaired after motor learning and paired-associative stimulation, Eur. J. Neurosci., 2012, 35, 975-986

[89] Belvisi D., Suppa A., Marsili L., Di Stasio F., Parvez A.K., Agostino R., et al., Abnormal experimentally- and behaviorally-induced LTP-like plasticity in focal hand dystonia, Exp. Neurol., 2013, 240, 64-74

[90] Hubsch C., Roze E., Popa T., Russo M., Balachandran A., Pradeep S., et al., Defective cerebellar control of cortical plasticity in writer's cramp, Brain, 2013, 136, 2050-2062

[91] Fiorio M., Tinazzi M., Aglioti S.M., Selective impairment of hand mental rotation in patients with focal hand dystonia, Brain, 2006, 129, 47-54

[92] Fiorio M., Tinazzi M., Ionta S., Fiaschi A., Moretto G., Edwards M.J., et al., Mental rotation of body parts and non-corporeal objects in patients with idiopathic cervical dystonia, Neuropsychologia, 2007, $45,2346-2354$

[93] Stoodley C.J., Schmahmann J.D., Evidence for topographic organization in the cerebellum of motor control versus cognitive and affective processing, Cortex, 2010, 46, 831-844

[94] Stoodley C.J., The cerebellum and cognition: evidence from functional imaging studies, Cerebellum, 2012, 11, 352-365

[95] Grimaldi G., Argyropoulos G.P., Boehringer A., Celnik P., Edwards M.J., Ferrucci R., et al., Non-invasive cerebellar stimulation - a consensus paper, Cerebellum, 2013, [Epub ahead of print], doi: 10.1007/s12311013-0514-7

[96] Bolognini N., Pascual-Leone A., Fregni F., Using non-invasive brain stimulation to augment motor training-induced plasticity, J. Neuroeng. Rehabil., 2009, 6, 8

[97] Monte-Silva K., Kuo M.F., Liebetanz D., Paulus W., Nitsche M.A., Shaping the optimal repetition interval for cathodal transcranial direct current stimulation (tDCS), J. Neurophysiol., 2010, 103, 17351740 